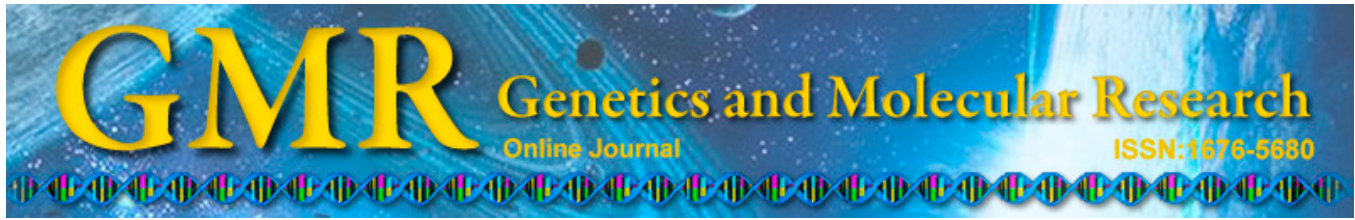

\title{
Clinical and cytogenetic results of a series of amniocentesis cases from Northeast China: a report of 2500 cases
}

\author{
N. An, L.L. Li, R.X. Wang, L.L. Li, J.M. Yue and R.Z. Liu \\ Center for Reproductive Medicine of the First Bethune Hospital of Jilin University, \\ Changchun, Jilin, China \\ Corresponding author: R.Z. Liu \\ E-mail: 1rz410@126.com
}

Genet. Mol. Res. 14 (4): 15660-15667 (2015)

Received July 1, 2015

Accepted September 22, 2015

Published December 1, 2015

DOI http://dx.doi.org/10.4238/2015.December.1.18

\begin{abstract}
The aims of this study were to demonstrate the clinical and cytogenetic results of amniocentesis (AS) cases in Northeast China, to compare the incidence of different kinds of chromosomal abnormalities, and to study the association between the detection rate of chromosomal abnormalities and different indications for prenatal diagnosis. Cytogenetic analysis was performed on long-term tissue cultures of 2500 second-trimester amniotic fluid samples. The most common indication for genetic AS was abnormal maternal serumscreening test $(69.56 \%)$, followed by advanced maternal age (15.04\%). Chromosomal abnormality was detected in $206(8.24 \%)$ of the 2500 samples. The detection rate of abnormal karyotypes was $62.5 \%$ in the group in which one member of the couple was a carrier of a chromosome abnormality; in the group having a positive result from noninvasive prenatal testing, the frequency was $50 \%$. To determine the origin of fetal chromosome abnormal karyotype, 45 fetuses were analyzed. Of these, 20 were found to be de novo abnormalities and 25 were familial. The frequency and proportion of abnormal karyotypes varied substantially across different maternal AS indications. Knowing
\end{abstract}


the origin and type of chromosomal abnormality would help determine termination or continuation of the pregnancy.

Key words: Amniocentesis; Chromosomal abnormality; Prenatal diagnosis; Genetic counseling

\section{INTRODUCTION}

In China, an increased number of pregnancies in women aged 35 years and older and positive results of maternal serum screening have markedly increased the demand for prenatal testing and genetic counseling. Prenatal diagnosis, particularly amniocentesis (AS), was introduced into clinical practice in the 1970s as the mid-trimester diagnostic investigation of choice (Daniilidis et al., 2008). It is currently the most commonly used invasive prenatal diagnostic method. At present, the importance of AS has been emphasized due to advances in screening maternal serum and ultrasonography, increasing awareness of congenital anomalies due to environmental pollution, and increasing maternal age (Han et al., 2008). In this study, we analyzed the clinical indications and cytogenetic analyses of 2500 second-trimester AS cases from our center with prenatal diagnosis and investigated the frequencies of different clinical indications and abnormal results.

\section{MATERIAL AND METHODS}

This study included a retrospective evaluation of the clinical indications and the cytogenetic results of 2500 second-trimester AS cases in the First Bethune Hospital of Jilin University between February 2011 and April 2014. The median age of the mothers was 30.5 (range 20-41 years). The indications of AS for cytogenetic analysis included: 1) increased risk in maternal serum screening (MSS); 2) advanced maternal age (AMA) ( $\geq 35$ years at the expected time of delivery); 3) both MSS and AMA were considered; 4) abnormal ultrasonographic findings; 5) a history of intrauterine fetal death or aborted fetuses; 6) one parent was a carrier of a chromosomal abnormality; or 7) positive results from noninvasive prenatal testing (NIPT). All of the patients with any of these indications were provided with genetic counseling about the possible risk of a chromosomal abnormality in the presence of their associated risk factors and were informed about the clinical significance of these results by the genetic counselor before deciding on pregnancy termination or continuation.

AS was performed between 16 and 18 weeks of gestation, when the procedure is safest (Alfirevic et al., 2003). Each patient signed an informed consent prior to the procedure. Under ultrasonographic guidance, the insertion angle and direction of the needle were determined at the best point where fluent amniotic fluid and limbs of the fetus were observed, avoiding the placenta and umbilical cord. An amniotic fluid sample $(25 \mathrm{~mL})$ was collected and transferred directly to the laboratory for culture, discarding the first $1-2 \mathrm{~mL}$ of the sample. After centrifugation at $1500 \mathrm{rpm}$ for $6 \mathrm{~min}$, the supernatant was discarded, and $5 \mathrm{~mL}$ culture medium (GIBCO AmnioMAX-II complete, USA) was inoculated with 1-1.5 mL cell suspension. The culture was maintained for $6-7$ days at $37^{\circ} \mathrm{C}$ and $5 \% \mathrm{CO}_{2}$ until cell growth was observed under an inverted microscope. G-banding was performed for chromosomal sample preparation, following the ISCN 2009 standard to analyze the karyotypes. The results of the cytogenetic analysis were divided into three groups as: 1) numerical chromosomal abnormalities; 2) structural chromosomal abnormalities; and 3) polymorphisms. 


\section{RESULTS}

Among the 2500 gravidas who underwent AS, the most common indication for AS in our center was abnormal MSS (69.56\%), followed by AMA (15.04\%), both MSS positive and AMA (8.92\%), abnormal US findings (3.48\%), a history of intrauterine fetal death or aborted fetuses $(2.52 \%)$, parent chromosome abnormality carriers $(0.32 \%)$, and others $(0.16 \%)$. The frequency of chromosomal abnormalities was significantly high in the group of couples with a history of intrauterine fetal death or aborted fetuses $(62.5 \%)$, followed by the group having positive results from noninvasive prenatal testing $(50 \%)$, and abnormal ultrasonographic findings $(19.54 \%)$. When both MSS and AMA were considered, the frequency of fetal chromosomal abnormalities was $14.35 \%$, if only MSS or AMA was considered, the frequency of fetal chromosomal abnormalities was 6.73 and $6.38 \%$, respectively. As a result, the frequency of fetal chromosomal abnormalities was significantly high when both MSS and AMA were considered for performance of genetic testing (Table 1).

Table 1. Distribution of chromosomal abnormalities according to the clinical indications for amniocentesis.

\begin{tabular}{lcc}
\hline Indication for amniocentesis & Proportion & Detection rate of abnormal karyotypes \\
\hline Maternal serum screening (MSS) positive & $69.56 \%(1739 / 2500)$ & $6.73 \%(117 / 1739)$ \\
Advanced maternal age (AMA) & $15.04 \%(376 / 2500)$ & $6.38 \%(24 / 376)$ \\
Both AMA and MSS & $8.92 \%(223 / 2500)$ & $14.35 \%(32 / 223)$ \\
Abnormal ultrasonographic findings (US) & $3.48 \%(87 / 2500)$ & $19.54 \%(17 / 87)$ \\
A history of intrauterine fetal death or aborted fetuses & $2.52 \%(63 / 2500)$ & $14.29 \%(9 / 63)$ \\
One of the couple carries chromosomal abnormality carrier & $0.32 \%(8 / 2500)$ & $62.50 \%(5 / 8)$ \\
Noninvasive prenatal testing (NIPT) positive & $0.16 \%(4 / 2500)$ & $50.00 \%(2 / 4)$ \\
Total & $100.00 \%(2500 / 2500)$ & $8.24 \%(206 / 2500)$ \\
\hline
\end{tabular}

Overall, 2500 cases were selected for inclusion in this study. Chromosomal abnormalities were detected in 206 of the 2500 cases (Table 2). Among these, $33.00 \%$ (68/206) were numerical abnormalities, $19.90 \%$ (41/206) were structural abnormalities, and $47.09 \%(97 / 206)$ were polymorphic variants (clinically insignificant heterochromatin area chromosomal aberrations that were not expected to have any phenotypic effects). The most frequently observed polymorphism was an increase in the heterochromatin region of chromosome 1, which was similar to the findings of Ocak et al. (2014), followed by a pericentric inversion on chromosome 9 .

Table 2. Types and proportion of chromosomal abnormalities detected in prenatal diagnosis.

\begin{tabular}{lrr}
\hline Types & Number & Proportion \\
\hline Numerical abnormalities & 68 & $33.00 \%(68 / 206)$ \\
Trisomy 21 & 41 & $19.90 \%(41 / 206)$ \\
Trisomy 18 & 13 & $6.31 \%(13 / 206)$ \\
Trisomy 13 & 1 & $0.97 \%(1 / 206)$ \\
$45, X$ & 3 & $1.46 \%(3 / 206)$ \\
47, XXX & 3 & $1.46 \%(3 / 206)$ \\
Mosaicism & 6 & $2.91 \%(6 / 206)$ \\
Structural abnormalities & 41 & $19.90 \%(41 / 206)$ \\
Inversion* & 27 & $13.11 \%(27 / 206)$ \\
Translocation & 15 & $7.28 \%(15 / 206)$ \\
Polymorphic variants & 97 & $47.09 \%(97 / 206)$ \\
Total & 206 & $100.00 \%(206 / 206)$ \\
\hline
\end{tabular}

*Inversion is exclusive of inv(9). 
The primary abnormal karyotype was trisomy 21 (exclusive of polymorphic variants), except for the groups in which one member of the couple carried a chromosomal abnormality or there was a history of intrauterine fetal death or spontaneous abortion. The group having positive results of NIPT had the highest detection rate of chromosomal numerical abnormalities $(50 \%$; $2 / 4$ ), followed by the abnormal ultrasonographic findings group (13.80\%) and the group positive for both AMA and MSS positive (7.62\%). The group in which one member of the couple carried a chromosomal abnormality had the highest detection rate of structural abnormalities $(62.50 \%)$, followed by the group having a history of intrauterine fetal death or spontaneous abortions (4.76\%). Additionally, the groups in which members were positive for MSS or had a history of miscarriage or intrauterine fetal death $(7.94 \%)$ had a high detection rate of polymorphic variants.

The parents of 45 fetuses were analyzed to determine the origin of abnormal karyotypes. During the analysis, 20 (44.44\%) de novo abnormalities were found, most of which were numerical abnormalities, and $25(55.56 \%)$ familial abnormalities were observed. Among the 25 familial cases, there were 21 cases with chromosomal polymorphisms, 3 cases with balanced translocations, and 1 case with inversion. Of the cases of abnormal fetal karyotypes, 13 (52\%) fetuses showed the same karyotype as that of their fathers, and $12(48 \%)$ had karyotypes identical to that of their mothers. Additionally, for all cases with chromosomal abnormalities identified via AS, the parents were informed about the genetic defect found and the consequences for the fetus were discussed in each case. In cases with a numerical abnormality, the option to terminate the pregnancy was provided to the parents, and in cases with structural abnormality and polymorphism, the parents were provided with the option to continue the pregnancy (Table 3 ).

Table 3. Lymphocyte karyotype analysis of the couples.

\begin{tabular}{|c|c|c|c|c|}
\hline Fetal karyotype & Father's karyotype & Mother's karyotype & Number $(\mathrm{N})$ & Outcome of pregnancy \\
\hline 46,XY,Yqh+ & 46,XY,Yqh+ & $46, \mathrm{XX}$ & 3 & Continuing \\
\hline $46, \mathrm{XN}, \operatorname{inv}(9)(\mathrm{p} 11 \mathrm{q} 13)$ & 46,XY,inv(9)(p11q13) & $46, \mathrm{XX}$ & 4 & Continuing \\
\hline $46, \mathrm{XN}, 15 \mathrm{pstk}+$ & $46, \mathrm{XY}, 15$ pstk + & $46, X X$ & 1 & Continuing \\
\hline $46, \mathrm{XN}, 16 \mathrm{qh}+$ & $46, \mathrm{XY}, 16 \mathrm{qh}+$ & $46, X X$ & 2 & Continuing \\
\hline $46, \mathrm{XN}, 9 \mathrm{qh}+$ & $46, \mathrm{XY}, 9 \mathrm{qh}+$ & $46, X X$ & 1 & Continuing \\
\hline $46, \mathrm{XN}, \mathrm{t}(5 ; 20)(\mathrm{q} 13 ; \mathrm{q} 12)$ & $46, X Y, t(5 ; 20)(q 13 ; q 12)$ & $46, X X$ & 2 & Continuing \\
\hline $46, \mathrm{XN}, \operatorname{inv}(22)(\mathrm{p} 11 \mathrm{p} 12)$ & $46, X Y, \operatorname{inv}(22)(\mathrm{p} 11 \mathrm{p} 12)$ & $46, X X$ & 1 & Continuing \\
\hline $46, \mathrm{XN}, 9 \mathrm{qh}+$ & $46, X Y$ & $46, \mathrm{XX}, 9 \mathrm{qh}+$ & 2 & Continuing \\
\hline $46, \mathrm{XN}, 16 \mathrm{qh}+$ & $46, X Y$ & $46, \mathrm{XX}, 16 \mathrm{qh}+$ & 1 & Continuing \\
\hline $46, X N, \operatorname{der}(21 ; 22)(q 10 ; q 10)$ & $46, X Y$ & $45, \mathrm{XX}, \operatorname{der}(21 ; 22)(\mathrm{q} 10 ; \mathrm{q} 10)$ & 1 & Continuing \\
\hline $46, \mathrm{XN}, 15$ pstk + & $46, X Y$ & $46, \mathrm{XX}, 15 \mathrm{pstk}+$ & 1 & Continuing \\
\hline 46,inv(9)(p11q13) & $46, X Y$ & $46, X X, \operatorname{inv}(9)(p 11 q 13)$ & 2 & Continuing \\
\hline $46, \mathrm{XN}, 22$ pstk + & $46, X Y$ & $46, \mathrm{XX}, 22 \mathrm{pstk}+$ & 1 & Continuing \\
\hline $46, \mathrm{XN}, 13$ pstk- & $46, X Y$ & $46, \mathrm{XX}, 13$ pstk- & 2 & Continuing \\
\hline $46, \mathrm{XN}, 14$ pstk + & $46, X Y$ & $46, \mathrm{XX}, 14 \mathrm{pstk}+$ & 1 & Continuing \\
\hline $47, \mathrm{XN},+21$ & $46, X Y$ & $46, \mathrm{XX}$ & 9 & Termination \\
\hline $47, \mathrm{XN},+18$ & $46, X Y$ & $46, X X$ & 2 & Termination \\
\hline $45, X$ & $46, X Y$ & $46, X X$ & 3 & Termination \\
\hline $46, \mathrm{XN}, 15 \mathrm{pstk}+$ & $46, X Y$ & $46, \mathrm{XX}$ & 1 & Continuing \\
\hline $46, \mathrm{XN}, 16 \mathrm{qh}+$ & $46, X Y$ & $46, X X$ & 1 & Continuing \\
\hline $46, \mathrm{XN}, 21$ pstk + & $46, X Y$ & $46, X X$ & 3 & Continuing \\
\hline $46, \mathrm{XN}, 22$ pstk + & $46, X Y$ & $46, X X$ & 1 & Continuing \\
\hline Total & - & - & 45 & Continuing \\
\hline
\end{tabular}

\section{DISCUSSION}

In the 1980s, AS was used primarily for patients of advanced maternal age (at least 35 
years old). Up until now, reports from other countries have still shown that prenatal diagnosis of chromosomal disorders is performed mainly for gravidas of an advanced maternal age. For example, as reported in the results of a study of 7028 cases by Tseng et al. (2006), AMA was the most common indication for AS. In a larger study from Korea, which included 31,615 cases of mid-trimester AS, abnormal MSS results were reported to be the most common indications for AS since 1994, followed by AMA and abnormal ultrasonographic findings (Han et al., 2008). The same results were found in our study; increased risk in MSS was the most common indication for AS, followed by AMA. With advanced technology increasing the detection rate of MSS, abnormal MSS results have become a more frequent indication for invasive testing.

The frequency of abnormal karyotypes was highest in the group in which one member of the couple carried a chromosomal abnormality, followed by the groups having a positive result from noninvasive prenatal testing, abnormal ultrasonographic findings, and members with both MSS and AMA. A comparison of the incidence rate of fetal chromosomal abnormalities demonstrated that the group for which both abnormal MSS and AMA were indications considered for performing AS had an incidence rate that was approximately twice that of the group for whom AMA was the only indication. Our results support the opinion that, for a more precise analysis of fetal chromosomal abnormalities, prenatal genetic counseling along with serum screening, ultrasound screening, a history of fetal chromosomal abnormalities, or the obstetrical history of the pregnant woman is necessary rather than a simple reference to maternal age alone (Kim et al., 2013). Therefore, the advanced age of a pregnant woman cannot be considered as a sole indication for AS, as the recent American College of Obstetrics and Gynecology (ACOG) Practice Bulletin recommended that screening and invasive diagnostic testing for aneuploidy should be available to all women who present for prenatal care before 20 weeks of gestation regardless of maternal age. In China, maternal age is still recommended as the single risk parameter to offer second trimester genetic AS. This is based on the health law of the government in China; however, most AMA participants knew that their serum screening risk estimate was 'normal' but still considered themselves at high risk due to their age. As childbearing is increasingly delayed, choosing an effective prenatal screening and diagnosis strategy is a practical social problem for China, and one feasible solution is to offer the screening to AMA women in an attempt to decrease the numbers undergoing genetic AS (Qi et al., 2013).

The results of prenatal diagnosis of AS, consisting of various numbers of cases, have revealed that the incidence of fetal chromosomal abnormalities ranges between 1.0 and 6.7\% (Simpson et al., 1976; Karaoguz et al., 2006; Tseng et al., 2006), higher than the incidence of chromosomal disorders in live newborns (0.5\%; Cerrillo Hinojosa et al., 2009). However, this study found that 206 of 2500 cases (8.24\%) had chromosomal abnormalities, a result that was higher than previously reported. This may have been caused by classifying polymorphic variants as a chromosome abnormality. Among the abnormalities, $33.00 \%$ were numeric abnormalities, $19.90 \%$ were structural abnormalities, and $47.09 \%$ were polymorphic variants. The most frequent numerical abnormality in all indications for AS was trisomy 21. Neagos et al. (2011) concluded that the percentage of the pregnancies with trisomy 21 was higher for the pregnant mothers who were 41-45 years old than for those who were 26-30 years old. Similar studies have reported that the risk for a 20 -year-old woman to have a child with Down's syndrome is about 1 in 2000 births, but it increases to approximately 1 in 40 births when the mother is 45 years of age. As approximately $55 \%$ of all Down's syndrome children are born to mothers over age 35 , it should be possible to halve the incidence of this 
syndrome by monitoring all pregnancies in this age group with AS (Crandall and Sparkes, 1973). We used a combination of methods for the detection of various syndromes. To confirm that the maternal serum screening was a critical step in detecting Down syndrome, maternal serum screening was provided to AMA women, which increased the detection rate of fetal chromosomal abnormalities.

Nakata et al. (2010) evaluated the trends in the choice of invasive prenatal diagnosis compared with the choice of first and second trimester prenatal serum screening for Down syndrome among patients referred for genetic counseling for AMA. They found that improved prenatal screening tests and increased availability of screening for AMA patients led to a steady decline in the choice of invasive testing from 2001 to 2007. Our results indicated that the group having positive results from NIPT had the highest detection rate of chromosomal numerical abnormalities $(50 \% ; 2 / 4)$, supporting previous findings that NIPT has been shown to be highly accurate in the detection of common fetal autosomal trisomies, especially T21 (Benn et al., 2013). In addition, ultrasonographic findings also play an important role in prenatal diagnosis of chromosomal abnormalities, because chromosome abnormalities, especially numerical abnormality, have characteristic sonographic signs. Nevertheless, prior to undertaking invasive prenatal diagnostic testing following non-invasive screening, prospective parents should be counseled concerning the possible presence of a fetal chromosomal abnormality other than trisomy 21 , and, in such cases, further genetic evaluation and testing may be required.

In all AS cases with a chromosome abnormality, the parents were informed about the genetic defect found and the consequences for the fetus were discussed for each case. In cases with numerical abnormalities, the parents' option was to terminate the pregnancy. In cases with structural abnormalities or polymorphic variants, the parents were given the option to continue the pregnancy, because there were no severe clinical manifestations.

In this study, we found 27 cases with inversions and 15 cases with translocations. Among the 15 cases with translocations, there were 5 cases of unbalanced chromosomal aberrations. These structural unbalanced chromosomal aberrations may lead to phenotypic anomalies that can vary from minor dysmorphism to severe malformations affecting the vital prognosis of the fetus. For these cases, the management of the pregnancy implies a comprehensive ultrasound evaluation followed by genetic counseling in order to inform the parents about the pregnancy risk, the possible fetal development, and the consequences of the genetic defect identified. Depending on the severity of the fetal malformations, the parents can choose to terminate the pregnancy or to have the child.

For the 10 cases of balanced chromosomal aberrations, additional tests are required in order to correctly characterize the anomaly. These are especially necessary for de novo chromosomal rearrangements. In addition, the incidence of polymorphic variants was $3.88 \%$, twice as high as the incidence in the general population. The most frequently observed polymorphisms were $1 \mathrm{qh}+$, inv(9), and $16 \mathrm{qh}+$. In recent years, the possible clinical importance of polymorphisms in the heterochromatin region of chromosomes has been reported, particularly their possible association with reproductive failure and recurrent spontaneous miscarriages. The abnormality of body and short arm of polymorphism of the D/G group directed effects on the cell division, resulting in embryonic developmental disorders and causing sterility or abortion and so on (Shaw-Smith et al., 2004). After excluding variant forms, inversions were found in about 1 of 100 cases, a frequency significantly higher than that reported by Forabosco et al. (2009) which may be influenced by differences in ethnic backgrounds. 
In order to confirm whether the fetal chromosomal abnormality was inherited or de novo, all parents were informed about the importance of testing their genetic material. However, peripheral blood lymphocytes for karyotype analysis were only collected from 45 parents; the rest of the couples refused further investigations or did not present for blood sampling. As described in Table 3, 20 chromosomal abnormalities were found to be de novo and 25 were familial. Among the 20 de novo abnormalities, most of them were numerical abnormalities. Among the 25 cases, the fetal karyotypes were the same as one of their parents, and the most common karyotype was inv(9), including 4 paternal cases and 2 maternal cases. In addition, for the cases with numerical abnormality and unbalanced chromosomal defects, the parents were informed about the genetic defect found, and the consequences for the fetus were discussed for each case. In the majority of the cases, the parents chose to terminate the pregnancy. The risk of phenotypic abnormality is very low when balanced chromosome rearrangement is inherited from a phenotypically normal carrier parent (Caron et al., 1999); however, some parents may choose to terminate the pregnancy voluntarily if they have concerns regarding future fertility problems for their child.

Polymorphisms are responsible for most of the genetic variations in populations and are generally not associated with clinical diseases, so in these cases, parents can consider continuing the pregnancy. However, the interpretation and counseling on de novo chromosomal rearrangements in prenatal diagnosis, compared with familial inheritance, is more difficult (Warburton, 1991). For these cases, we further confirmed the rearrangements using new molecular techniques in order to elucidate the characteristics of the chromosomes more clearly.

In conclusion, abnormal karyotype composition varies according to different maternal AS indications. Our data could offer a database for proper prenatal genetic counseling of pregnant women and their partner, and for future pregnancies in China. There is a variety of abnormal karyotypes in the second trimester of pregnancy. As the risk of fetal malformation is related to the kind of abnormal karyotype, an accurate characterization of the fetal chromosomal defects has implications on the couple's decision regarding the continuation of the pregnancy and brings important information for the future reproductive options in order to give birth to a healthy baby. The present study provides informative data for counseling of the women of Northeast China and their partners. However, more cases should be examined further to obtain greater accuracy in predicting the rates of each chromosomal abnormality at mid-trimester AS.

\section{Conflicts of interest}

The authors declare no conflict of interest.

\section{ACKNOWLEDGMENTS}

We express our deepest gratitude to all of the staff of the Genetics Laboratory and Andrology Laboratory for their excellent work and advice to make this research possible. Research supported by the National Population and Family Planning Commission of China (\#2011-GJKJS-07). 


\section{REFERENCES}

Alfirevic Z, Sundberg K and Brigham S (2003). Amniocentesis and chorionic villus sampling for prenatal diagnosis. Cochrane Database Syst Rev. CD003252.

Benn P, Borell A, Chiu R, Cuckle H, et al. (2013). Position statement from the Aneuploidy Screening Committee on behalf of the Board of the International Society for Prenatal Diagnosis. Prenat. Diagn. 33: 622-629.

Caron L, Tihy F and Dallaire L (1999). Frequencies of chromosomal abnormalities at amniocentesis: over 20 years of cytogenetic analyses in one laboratory. Am. J. Med. Genet. 82: 149-154.

Cerrillo Hinojosa M, Yerena de Vega MC, Gonzalez Panzzi ME, Godoy H, et al. (2009). Genetic amniocentesis in highrisk populations. Experience in 3081 cases. Ginecol. Obstet. Mex. 77: 173-182.

Crandall BF and Sparkes RS (1973). Prenatal diagnosis of genetic disorders by amniocentesis. Calif. Med. 119: 1-6.

Daniilidis A, Karydas H, Zournatzi V, Tantanasis T, et al. (2008). A four-year retrospective study of amniocentesis: one centre experience. Hippokratia 12: 113-115.

Forabosco A, Percesepe A and Santucci S (2009). Incidence of non-age-dependent chromosomal abnormalities: a population-based study on 88965 amniocenteses. Eur. J. Hum. Genet. 17: 897-903.

Han SH, An JW, Jeong GY, Yoon HR, et al. (2008). Clinical and cytogenetic finding on 31,615 mid-trimester amniocenteses. Korean J. Lab. Med. 28: 378-385.

Karaoguz MY, Bal F, Yakut T, Ercelen NO, et al. (2006). Cytogenetic results of amniocentesis materials: incidence of abnormal karyotypes in the Turkish collaborative study. Genet. Couns. 17: 219-230.

Kim YJ, Lee JE, Kim SH, Shim SS, et al. (2013). Maternal age-specific rates of fetal chromosomal abnormalities in Korean pregnant women of advanced maternal age. Obstet. Gynecol. Sci. 56: 160-166.

Nakata N, Wang Y and Bhatt S (2010). Trends in prenatal screening and diagnostic testing among women referred for advanced maternal age. Prenat Diagn. 30: 198-206.

Neagos D, Cretu R, Sfetea RC and Bohiltea LC (2011). The importance of screening and prenatal diagnosis in the identification of the numerical chromosomal abnormalities. Maedica 6: 179-184.

Ocak Z, Özlü T, Yazıcıoglu HF, Özyurt O, et al. (2014). Clinical and cytogenetic results of a large series of amniocentesis cases from Turkey: report of 6124 cases. J. Obstet. Gynaecol. Res. 40: 139-146.

Qi QW, Jiang YL, Zhou XY, Liu JT, et al. (2013). Genetic counseling, prenatal screening and diagnosis of Down syndrome in the second trimester in women of advanced maternal age: a prospective study. Chin. Med. J. 126: 2007-2010.

Shaw-Smith C, Rendon R, Rickman L, Rio M, et al. (2004). Microarray based comparative genomic hybridisation (array$\mathrm{CGH}$ ) detects submicroscopic chromosomal deletions and duplications in patients with learning disability/mental retardation and dysmorphic features. J. Med. Genet. 41: 241-248.

Simpson NE, Dallaire L, Miller JR, Siminovich L, et al. (1976). Prenatal diagnosis of genetic disease in Canada: report of a collaborative study. Can. Med. Assoc. J. 23: 739-748.

Tseng JJ, Chou MM, Lo FC, Lai HY, et al. (2006). Detection of chromosome aberrations in the second trimester using genetic amniocentesis: experience during 1995-2004. Taiwan J. Obstet. Gynecol. 45: 39-41.

Warburton D (1991). De novo balanced chromosome rearrangements and extra marker chromosomes identified at prenatal diagnosis: clinical significance and distribution of breakpoints. Am. J. Hum. Genet. 49: 995-1013. 